\title{
Meagerness of Lipschitz Functions
}

\author{
$\mathrm{Yu}-$ Lin $\mathrm{Chou}^{*}$
}

\begin{abstract}
We show that the class of Lipschitz real-valued functions on a compact set $K$ in $\mathbb{R}$ is meager in $C(K)$ with respect to the supremum metric. As these Lipschitz functions are dense in $C(K)$, this result complements the classical surprising result for the meagerness of somewhere differentiable functions in $C([0,1])$.
\end{abstract}

MSC 2020: 26A16; 26A21; 26A15

Keywords: Baire categories; compact spaces; continuous functions; Lipschitz functions; meager sets

Throughout, every function is taken as real-valued; every space of continuous functions on a compact space is equipped with the supremum metric. For reference: A Lipschitz function on a subset of $\mathbb{R}$ is by definition precisely a function $f$ such that $|f(x)-f(y)| \leq M|x-y|$ for some real $M \geq 0$ and all $x, y$ in the domain; a meager set in a topological space is by definition precisely a countable union of nowhere dense sets, i.e. a countable union of sets whose closure has empty interior.

It is well-known that somewhere differentiable functions in $C([0,1])$ are "scarce" in the sense that they form a meager set, which is surprising in contrast with both the denseness of differentiable functions in $C([0,1])$ and a belief known ([1]) to be once generally held.

Also unexpected is the Morgenstern's result that somewhere (real-)analytic functions in $C^{\infty}([0,1])$ form a meager set.

Since Lipschitz functions on a compact set $K$ in $\mathbb{R}$ are dense in $C(K)$, it would be interesting to know if Lipschitz functions in $C(K)$ are also meager. The question is affirmed by the following

Theorem 1. If $K \subset \mathbb{R}$ is compact, then the class of Lipschitz functions $K \rightarrow \mathbb{R}$ is meager in $C(K)$.

${ }^{*}$ Institute of Statistics, National Tsing Hua University, Hsinchu 30013, Taiwan, R.O.C.; Email: y.l.chou@gapp.nthu.edu.tw 
Proof. Fix any $x \in K$, and let

$$
A_{r}:=\{f \in C(K)|| f(x)-f(y)|\leq r| x-y \mid \text { for all } y \in K\}
$$

for all rational $r \geq 0$.

Denote by $C_{L}(K)$ the class of Lipschitz functions $K \rightarrow \mathbb{R}$. Evidently, if $f \in C_{L}(K)$ then $f \in A_{r}$ for some rational $r \geq 0$; in other words, we have

$$
C_{L}(K) \subset \bigcup_{r \in \mathbb{Q} \cap[0,+\infty[} A_{r}
$$

Denote by $d$ the supremum metric of $C(K)$. We claim that every $A_{r}$ is closed in $C(K)$. Indeed, we have

$$
A_{r}=\bigcap_{y \in K}\{f \in C(K)|| f(x)-f(y)|\leq r| x-y \mid\}=: \bigcap_{y \in K} A_{r, y}
$$

for all $r$. If $f \in A_{r, y}^{c}$, so that $|f(x)-f(y)|>r|x-y|$, we choose

$$
\varepsilon:=\frac{1}{2}(|f(x)-f(y)|-r|x-y|) .
$$

Then, since $|g(x)-g(y)|>|f(x)-f(y)|-2 d(f, g)$ for all $g \in C(K)$, it holds that $d(f, g)<\varepsilon$ implies $g \in A_{r, y}^{c}$; the claim follows.

To show that every $A_{r}$ is nowhere dense, let $f \in A_{r}$. If $\varepsilon>0$, we consider the function

$$
g: z \mapsto\left(\frac{\varepsilon}{\sup _{z \in K} z^{2}+1}\right) z^{2}+f(z)=: a z^{2}+f(z)
$$

on $K$. Then $d(f, g)<\varepsilon$. But since $|g(x)-g(y)|=|a(x-y)(x+y)+f(x)-f(y)|$ for all $y \in K$, if $g \in A_{r}$ then by triangle inequality the function $y \mapsto a|x+y|$ is majorized everywhere by a constant; so we have $g \in A_{r}^{c}$, and the desired nowhere denseness of $A_{r}$ follows. Since a subset of a meager set is meager, the proof is complete.

Remark. The proof of Theorem 1 indeed asserts more: Since $K$ is compact, upon choosing a countable dense subset $K_{0}$ of $K$ we see that the set

$$
\bigcup_{\substack{x \in K_{0} ; \\ r \in \mathbb{Q} \cap[0,+\infty[}}\{f \in C(K)|| f(x)-f(y)|\leq r| x-y \mid \text { for all } y \in K\}
$$

is also meager in $C(K)$.

It would be informative to add that, for suitable choices of $g$ (whenever possible), Theorem 1 is readily generalized for subclasses of the class of compact metric spaces.

For reference: a Baire space is by definition precisely a topological space where every countable intersection of open dense sets is dense. Then we have 
Corollary 1. If $K \subset \mathbb{R}$ is compact, and if $C_{L}(K)$ is the class of all Lipschitz functions $K \rightarrow \mathbb{R}$, then the interior of $C_{L}(K)$ in $C(K)$ is empty.

Proof. Since $C(K)$ is a complete metric space, it follows that $C(K)$ is Baire by a celebrated (standard) result of Baire's. Upon observing that, in any given topological space, if $A$ is nowhere dense then the complement $(\operatorname{cl}(A))^{c}$ of the closure of $A$ is dense, we see that every meager set in a Baire space has empty interior; this is also a result of Baire's. Therefore, as $C_{L}(K)$ is meager in $C(K)$ by Theorem 1, the desired conclusion is obtained.

\section{References}

[1] S.G. Krantz and H.R. Parks, The Geometry of Domains in Space, Springer, 1999. 